\title{
An Imported Confirmed Case of COVID-19 Vaccinated in Brazil — Shanghai Municipality, China, March 17, 2021
}

\author{
Hongrang Zhou ${ }^{1, \&, *}$; Xiaoling Wang, ${ }^{2, \&}$; Guifu $\mathrm{Li}^{1}$; Sen Wang'; Xiaoming Wang ${ }^{3}$; Yueqin Wang'; \\ Changpo Lin'; Jianguo Tian'; Junfeng Pan'; Ruifang Xu'; Ning Xiao,
}

On March 17, 2021, a 42-year-old male returning from Brazil tested positive by Qingpu CDC for coronavirus disease 2019 (COVID-19) during medical observation at a designated isolation point. He was then sent to the Shanghai Public Health Clinical Center (SHPHCC) and confirmed again, and because he demonstrated no abnormal clinical signs (until the infection was controlled), he was diagnosed as an asymptomatic infection.

According to an epidemiological investigation of this patient, the timeline of this patient's relevant history, from COVID-19 vaccination to release from medical observation, was shown in Figure 1. The patient had completed two doses of COVID-19 vaccination (Beijing Institute of Biological Products Co. LTD) in Brazil on September 7, 2020, and October 3, 2020. In addition, he had self-tested using an antibody kit on October 24, while the test results were negative. The patient maintained his normal work and living conditions during his stay in Brazil until January 4, 2021, when his test results of $\operatorname{IgM}$ and $\operatorname{IgG}$ of COVID-19 both returned positive. Since the conditions for returning to China were not met, the patient self-isolated at home until March 9, 2021. Because administration of the inactivated vaccine is followed by the production of $\operatorname{IgM}$ and $\operatorname{IgG}$ in the body, the policy on returning to China has since been adjusted.

On March 10, 2021 (GMT-3), the patient departed from Sao Paulo International Airport in Brazil and arrived at Shanghai Pudong International Airport on March $12(\mathrm{GMT}+8)$. The patient was sent to the corresponding isolation hotel. From January 4 to March 15, 2021, all tests [including nucleic acid tests and computed tomography (CT) tests] were normal, except for the results monitoring IgM and IgG, which were all positive. Subsequently, on March 16, due to two confirmed patients being on the same flight, he was considered a potential close contact and was sent to a designated isolation point for close contacts to receive medical isolation and observation. However, the nucleic acid test results of the nasopharyngeal swab of the patient were then positive on March 17 and were further confirmed at the SHPHCC. The patient recovered on March 28 and was then sent to a medical isolation point for another 14-day quarantine observation. With no abnormalities during the quarantine period, the patient was finally discharged from isolation on April 11.

Though the self-test results of the patient conducted on October 24, 2020 were negative, these results may be related to differences in the vaccines (1). This would likely be reflected in the patient still having a risk of COVID-19 infection during this period. In addition, the possibility of false negatives in the self-test could not be excluded. However, during the home isolation period of the patient in Brazil, the patient's serum antibody test results returned positive. IgM maintained a high titer for a long time, while the IgG titer gradually decreased, as shown in Figure 2 (according to

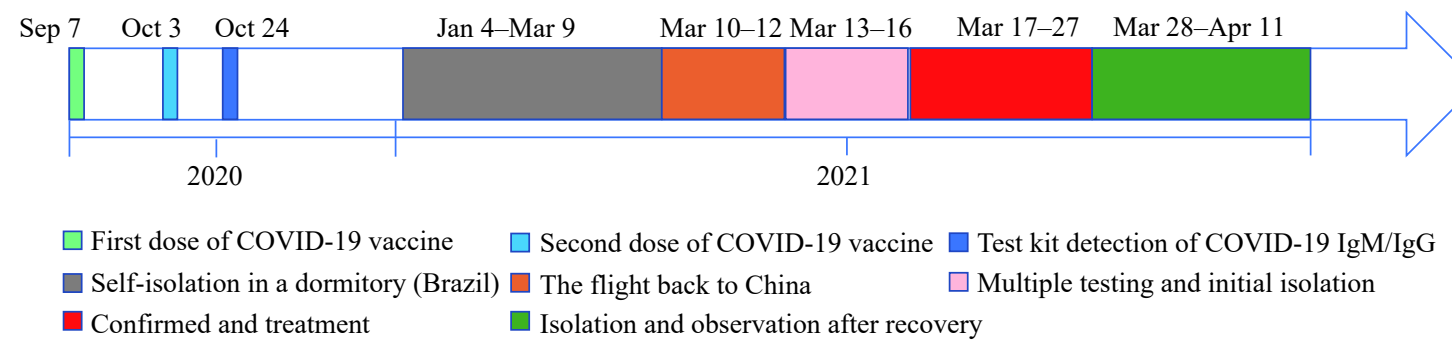

FIGURE 1. Timeline of relevant history of the patient in Brazil and China from September 7, 2020 to April 11, 2021. 


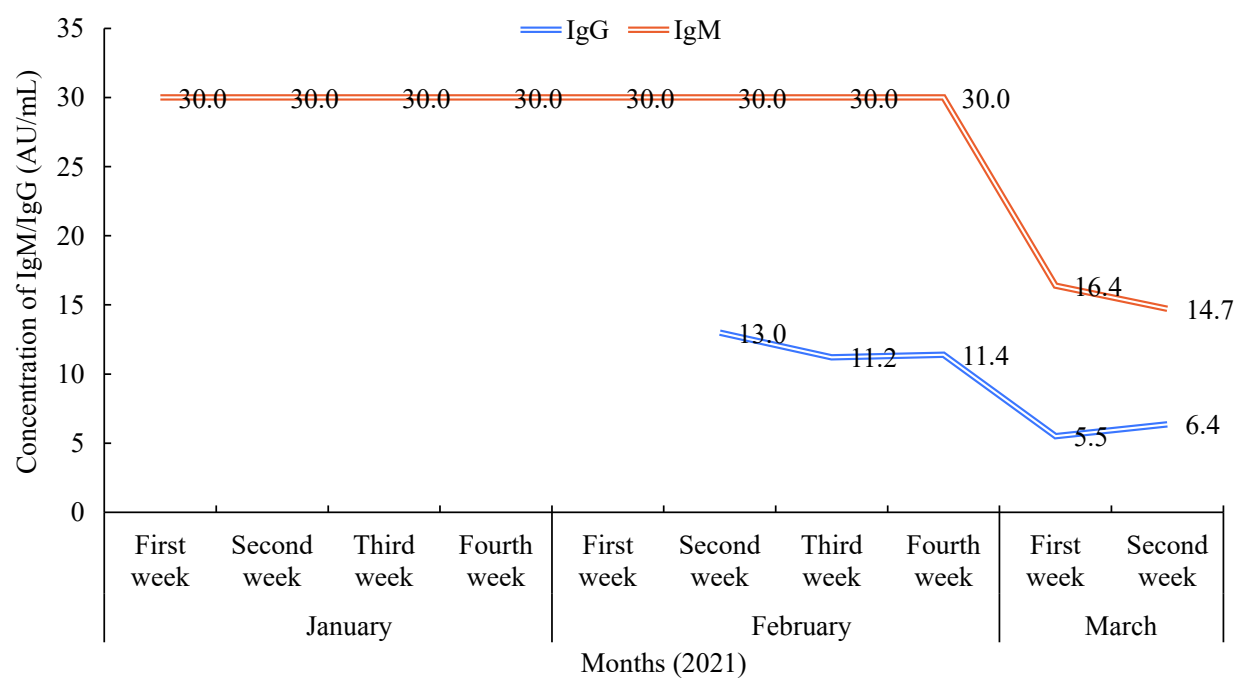

FIGURE 2. From January 4 to March 9,2021 , the changes in IgM/lgG titer of the patient after antibody testing in designated local hospitals selected by the Chinese Embassy in Brazil.

the data provided by the patient and part of the test report). Moreover, the antibody level of IgM from February to early March was about 2-3 times higher than that of $\operatorname{IgG}$ in each test result. As an asymptomatic infection, the changes of antibodies of the patient were different from those in related studies (2-3), so it is uncertain whether the antibody changes in this patient were caused by vaccination. Future research may yield clarification for this issue.

The patient in this investigation was vaccinated but still became infected with COVID-19. This implies that those receiving vaccines are still at a potential risk of infection under certain conditions. In addition, according to the official website of the US CDC, there are 9,425 people who have been vaccinated in the US that have become infected with COVID-19 (4), which demonstrates the continued, albeit diminished, risk of infection post-vaccination. However, vaccination is a process of stimulating the production of protective antibodies in the body. Usually there is no vaccine that can achieve $100 \%$ protection (5), meaning that the possibility of breakthrough infections still exists even after vaccination. Individual differences, vaccine failure during the vaccination process, COVID-19 mutant variants, and high-risk exposure behaviors may all contribute to breakthrough vaccine immunity (G). Therefore, the secondary infection post-vaccination is unsurprising. In the epidemiological investigation of this patient, we found that after COVID-19 vaccination, he had a history of suspected exposure to COVID-19 during his work in Brazil and during his journey returning to China. This may be the way that the patient was infected with COVID-19. Individual differences and other factors cannot be ruled out.

People who have completed a full course of vaccination are highly recommended to remain cautious and take personal protective measures such as wearing masks and maintaining appropriate social distance to protect themselves and others ( 7 ), regardless of whether vaccination has been completed or not. Before achieving herd immunity, these continued measures are essential to protect the health of individuals and the public.

doi: $10.46234 / \mathrm{ccdcw} 2021.140$

\# Corresponding authors: Hongrang Zhou, hrzhou0223@foxmail.com; Ning Xiao, xiaoning@nipd.chinacdc.cn.

\footnotetext{
${ }^{1}$ Qingpu District Center for Disease Control and Prevention, Shanghai, China; ${ }^{2}$ National Institute of Parasitic Diseases, Chinese Center for Disease Control and Prevention; Chinese Center for Tropical Diseases Research; WHO Collaborating Centre for Tropical Diseases; National Center for International Research on Tropical Diseases, Ministry of Science and Technology; Key Laboratory of Parasitic and Vector Biology, National Health Commission of China; The School of Global Health, Chinese Center for Tropical Diseases Research, Shanghai Jiao Tong University School of Medicine; Huangpu, Shanghai, China; ${ }^{3}$ Program in Public Health, College of Health Sciences, University of California at Irvine, Irvine, USA.

\& Joint first authors.
}

Submitted: May 10, 2021; Accepted: June 11, 2021

\section{REFERENCES}

1. Xia SL, Zhang YT, Wang YX, Wang H, Yang YK, Gao GF, et al. Safety and immunogenicity of an inactivated SARS-CoV-2 vaccine, BBIBPCorV: a randomised, double-blind, placebo-controlled, phase $1 / 2$ trial. Lancet Infect Dis 2021;21(1):39 - 51. http://dx.doi.org/10.1016/S14733099(20)30831-8.

2. Lei Q, Li Y, Hou HY, Wang F, Ouyang ZQ, Zhang YD, et al. Antibody dynamics to SARS-CoV-2 in asymptomatic COVID-19 infections. 
Allergy 2021;76(2):551 - 61. http://dx.doi.org/10.1111/all.14622.

3. Feng X, Yin JM, Zhang JY, Hu YL, Ouyang YB, Qiao SB, et al. Longitudinal profiling of antibody response in patients with COVID-19 in a tertiary care hospital in Beijing, China. Front Immunol 2021;12:614436. http://dx.doi.org/10.3389/fimmu.2021.614436.

4. Florescu L, Rugina A, Temneanu OR, Paduraru DTA, Matei MC, Safta C, et al. Vaccination--collective responsibility or violation of rights? Rev Med Chir Soc Med Nat Iasi 2015;119(4):1098-105. https://pubmed.ncbi. nlm.nih.gov/26793855/

5. Centers for Disease Control and Prevention. COVID-19 breakthrough case investigations and reporting. 2021. https://www.cdc.gov/vaccines/ covid-19/health-departments/breakthrough-cases.html. [2021-4-19].

6. Ma CF, Xu ST, Yao YC, Yu PB, Xu Y, Wu R, et al. Outbreak reports: mild breakthrough infection in a healthcare professional working in the isolation area of a hospital designated for treating COVID-19 patients-Shaanxi Province, China, March, 2021. China CDC Wkly 2021;3(19):397 - 400. http://dx.doi.org/10.46234/ccdcw2021.094.

7. Zhou HR, Wang XL, Xu QF, Wang XM, Ma YD, Shi YR, et al. Outbreak reports: an investigation of a confirmed imported case of COVID-19 infected abroad-Qingpu District, Shanghai Municipality, China, November 15, 2020. China CDC Wkly 2021;3(10):207 - 10. http://dx.doi.org/10.46234/ccdcw2021.061. 